\title{
On the Crossover from BCS Superconductivity to Bose Condensation
}

\author{
R. MiCnas \\ Institute of Physics, A. Mickiewicz University \\ Umultowska 85, 61-614 Poznań, Poland
}

(Received December 17, 2001)

\begin{abstract}
We outline a microscopic approach to the superconducting fluctuations and pairing correlations in the attractive Hubbard model above $T_{c}$, using the functional integral method. A crossover from BCS superconductivity to Bose condensation of preformed pairs is studied by constructing the appropriate Ginzburg-Landau functionals. The differences between the lattice and the continuum models are discussed. The case of quasi-two-dimensional superconductors as well as the model with non-local pairing interaction are also examined. The effects of Gaussian fluctuations of the order parameter are analyzed in the T-matrix approach, the self-consistent Hartree approach and finally within the Ginzburg-Landau theory. Similarities with a paramagnon theory of itinerant-electron magnetism are pointed out.
\end{abstract}

PACS numbers: $74.20 .-\mathrm{z}$

\section{Introduction}

The problem of a crossover from a weak coupling BCS superconductivity to Bose condensation (BC) of preformed local pairs has recently attracted great attention, stimulated by the experimental results concerning unusual properties of new high $T_{\mathrm{c}}$ superconductors as well as the discovery of Bose condensates in optically trapped dilute atomic gases. It has been established that high temperature superconductors (HTS) generally exhibit a low carrier density, small value of the Fermi energy $(\propto 0.1-0.3 \mathrm{eV})$, short coherence length $\xi_{0}$, and they are extreme type II superconductors with the Ginzburg-Landau (G-L) parameter $\kappa \gg 1$ [1-4]. Experimental studies of HTS and also other non-conventional superconductors indicate that the cuprate high $T_{c}$, bismuthates, fullerenes, and Chevrel phases belong 
to a unique group of superconductors characterized by high transition temperatures relative to the values of $n_{\mathrm{s}} / \mathrm{m}^{*}$. These materials have their $T_{\mathrm{c}}$ proportional to $T_{\mathrm{F}}$ (the Fermi temperature) or $T_{\mathrm{B}}$ (the Bose-Einstein condensation temperature) [2], with $T_{\mathrm{B}} \approx(3-30) T_{\mathrm{C}}$ and $T_{\mathrm{F}} \approx(10-100) T_{\mathrm{c}}$. Moreover, the existence of the pseudogap phase in the underdoped HTS is well experimentally established and it can be interpreted as a state with pairing without long-range phase coherence $[4,5]$. The above points show that these materials are not in a weak coupling BCS limit, but rather in the intermediate to extreme strong coupling regime.

One of the central questions regarding the physics of HTS is the understanding of the evolution from a weak coupling limit of large Cooper pairs to the strong coupling regime of small local pairs with increasing coupling constant and description of the intermediate (crossover) regime.

Several attempts to describe the crossover have employed the BCS mean field theory, generalized to allow a change in the chemical potential due to the pairing away from the weak coupling limit [6-11]. It has been shown that the BCS wave function for $T=0 \mathrm{~K}$ is a reasonable ansatz for any attraction strength. For the fermions in continuum, the controlling parameter is $\xi_{0} k_{\mathrm{F}}\left(\xi_{0}-\right.$ the coherence length, $k_{\mathrm{F}}$ - the Fermi momentum) and the BCS and BC limits correspond to $\xi_{0} k_{\mathrm{F}} \gg 1$ and $\xi_{0} k_{\mathrm{F}} \ll 1$, respectively. An extension to finite temperatures has been first proposed by Nozieres and Schmitt-Rink [9], who included single-pair fluctuations within a T-matrix approximation and found that $T_{\mathrm{c}}$ for the 3D continuum dilute fermion system evolves smoothly between the limits (see also Refs. [12-14]). Similar analysis has also been carried out for a 2D dilute attractive Fermi gas $[15,16]$.

In this paper we overview the functional integral approach to the crossover problem using mainly the Hubbard model with on-site attraction. The attractive Hubbard model is a generic model of superconductivity on a lattice which can describe the evolution from the BCS type superconductivity to a Bose condensation of real space pairs, with increasing coupling strength (for review see Refs. $[1,17,18])$. The layout of this paper is the following. In Sec. 2, we present application of the functional integration method to the negative $U$ Hubbard model. In Sec. 3, the crossover is studied by a detailed derivation of the Ginzburg-Landau theory in the weak attraction limit and the corresponding Ginzburg-Pitaevskii functional in the strong coupling regime. We discuss the differences between the lattice and continuum fermion models with $s$-wave attraction. The case of quasi-2D-superconductivity and the model with pair hopping interaction are also discussed. In Sec. 4, we discuss the Gaussian fluctuations and the T-matrix approach. The self-consistent approach to the superconducting fluctuations and pairing correlations is further discussed in Sec. 5, where a derivation of a self-consistent Gaussian theory is given. In Sec. 6 we analyze the amplitude fluctuations above $T_{\mathrm{c}}$ using the Ginzburg-Landau theory. 


\section{Functional integral approach}

Here we will outline the functional integral approach to the attractive Hubbard model. The Hamiltonian of the model can be split into two parts

$$
\begin{aligned}
& H=H_{0}+H_{1}, \\
& H_{0}=\sum_{i, j, \sigma} \hat{t}_{i j} c_{i \sigma}^{\dagger} c_{j \sigma}, \\
& H_{1}=-|U| \sum_{i} \rho_{i}^{\dagger} \rho_{i},
\end{aligned}
$$

where $\rho_{i}^{\dagger}=c_{i \uparrow}^{\dagger} c_{i \downarrow}^{\dagger}$, and $\hat{t}_{i j}=t_{i j}-\mu \delta_{i j} . t_{i j}$ is the transfer integral, $|U|$ - the on-site attraction and $\mu$ stands for the chemical potential. The grand partition function of the model (1) in the interaction representation with respect to $H_{0}$ is given by

$$
\begin{aligned}
Z= & \operatorname{Tr} \exp \left[-\beta\left(H_{0}+H_{1}\right)\right]=\operatorname{Tr}\left\{\mathrm{e}^{-\beta H_{0}} T_{\tau} \exp \left[-\int_{0}^{\beta} d \tau H_{1}(\tau)\right]\right\} \\
& =Z_{0}\left\langle T_{\tau} \exp \left[-\int_{0}^{\beta} d \tau H_{1}(\tau)\right]\right\rangle_{0},
\end{aligned}
$$

where $T_{\tau}$ is the "time" ordering operator, $H_{1}(\tau)=\exp \left(\tau H_{0}\right) H_{1} \exp \left(-\tau H_{0}\right)$, $Z_{0}=\operatorname{Tr} \mathrm{e}^{-\beta H_{0}},\langle\cdots\rangle_{0}=\operatorname{Tr}\left(\cdots \mathrm{e}^{-\beta H_{0}}\right) /\left(\operatorname{Tr} \mathrm{e}^{-\beta H_{0}}\right)$ and $\beta=\left(k_{\mathrm{B}} T\right)^{-1}$. The functional integral representation of $Z$ in terms of auxiliary fields $\Delta_{i}(\tau)$ is obtained in a standard manner by making use of the Hubbard-Stratonovich transformation [19]

$$
Z=Z_{0} \int D^{2} \Delta \exp \left(-\Psi\left[\Delta_{i}(\tau)\right]\right)
$$

where

$$
\begin{aligned}
& \Psi\left[\Delta_{i}(\tau)\right]=\sum_{i} \int_{0}^{\beta} d \tau\left|\Delta_{i}(\tau)\right|^{2} /|U|-\Omega[\Delta], \\
& \Omega[\Delta]=-\ln \left\langle T_{\tau}\left[\exp \left(\sum_{i} \int_{0}^{\beta} \mathrm{d} \tau\left[\Delta_{i}(\tau) \rho_{i}^{\dagger}(\tau)+\text { H.c. }\right]\right)\right]\right\rangle_{0},
\end{aligned}
$$

$D^{2} \Delta=D \Delta D \Delta^{*}$. Introducing the Nambu representation $\Psi_{i}^{\dagger}=\left(c_{i \uparrow}^{\dagger}, c_{i \downarrow}\right)$, one obtains for $\Omega[\Delta]$

$$
\Omega[\Delta]=-\ln \left\langle T_{\tau}\left[\exp \left(\sum_{i} \int_{0}^{\beta} \mathrm{d} \tau \Psi_{i}^{\dagger} \hat{\Delta}_{i}(\tau) \Psi_{i}(\tau)\right)\right]\right\rangle_{0},
$$


where $\hat{\Delta}_{i}=\left(\begin{array}{cc}0 & \Delta_{i} \\ \Delta_{i}^{*} & 0\end{array}\right)$. The matrix Green function

$$
\hat{\mathcal{G}}_{i, j}\left(\tau, \tau^{\prime}\right)=-\left\langle T_{\tau} \Psi_{i}^{\dagger}(\tau) \Psi_{j}\left(\tau^{\prime}\right)\right\rangle=\left(\begin{array}{cc}
G_{i j}\left(\tau, \tau^{\prime}\right) & F_{i j}\left(\tau, \tau^{\prime}\right) \\
F_{i j}^{\dagger}\left(\tau, \tau^{\prime}\right) & -G_{i j}\left(\tau, \tau^{\prime}\right)
\end{array}\right)
$$

where the field operators are given in the Heisenberg representation with respect to the Hamiltonian (1), can be evaluated within the functional integral scheme as follows:

$$
\hat{\mathcal{G}}_{i, j}\left(\tau, \tau^{\prime}\right)=\left\langle\hat{\mathcal{G}}_{i, j}\left(\tau, \tau^{\prime},\{\Delta\}\right)\right\rangle_{\Delta},
$$

where the average $\langle\cdots\rangle_{\Delta}=\int D^{2} \Delta \exp (-\Psi[\Delta])(\cdots) / \int D^{2} \Delta \exp (-\Psi[\Delta])$ denotes the functional average. The Green function $\hat{\mathcal{G}}_{i, j}\left(\tau, \tau^{\prime},\{\Delta\}\right)$ is just the Green function for electrons in the random fields $\Delta_{i}(\tau)$ and is given as

$$
\begin{gathered}
\hat{\mathcal{G}}_{i, j}\left(\tau, \tau^{\prime},\{\Delta\}\right)=-\frac{\left\langle T_{\tau} \Psi_{i}^{\dagger}(\tau) \Psi_{j}\left(\tau^{\prime}\right) S(\lambda)\right\rangle_{0}}{\langle S(\lambda)\rangle_{0}} \\
=-\left\langle T_{\tau} \Psi_{i}^{\dagger}(\tau) \Psi_{j}\left(\tau^{\prime}\right)\right\rangle_{H_{0}+H_{r}}, \\
S(\lambda)=T_{\tau} \exp \left(-\lambda \int_{0}^{\beta} \mathrm{d} \tau \Psi^{\dagger} \hat{\Delta}_{i}(\tau) \Psi_{i}(\tau)\right),
\end{gathered}
$$

$\langle\cdots\rangle_{H_{0}+H_{r}}$ denotes the average with the Hamiltonian $H_{0}+H_{r}$ with $H_{0}$ given by Eq. (1) and $H_{r}$ given below

$$
H_{r}=-\lambda \sum_{i}\left(\rho_{i}^{\dagger} \Delta_{i}(\tau)+\text { H.c. }\right)
$$

and $\lambda$ is a formal parameter being set to 1 at the end. This local Green's function satisfies the BCS equation in the presence of spatially and time varying local order parameter

$$
\sum_{l}\left(-\frac{\partial}{\partial \tau} \delta_{i l}-\hat{t}_{i l} \sigma_{z}-\lambda \hat{\Delta}_{i}(\tau)\right) \hat{\mathcal{G}}_{l j}\left(\tau, \tau^{\prime},\{\Delta\}\right)=\hat{1} \delta_{i j} \delta\left(\tau-\tau^{\prime}\right),
$$

where $\sigma_{z}$ is the Pauli matrix. An exact representation for the partition function can be given by

$Z=Z_{0} \int D^{2} \Delta \exp [-\Psi], \quad \Psi=\sum_{i} \int_{0}^{\beta} \mathrm{d} \tau\left|\Delta_{i}(\tau)\right|^{2} /|U|-\operatorname{Tr} \ln \left(1-\hat{\Delta} \hat{\mathcal{G}}_{0}\right)$,

where

$$
\hat{\mathcal{G}}_{0}^{-1}=\left(-\frac{\partial}{\partial \tau}-\hat{t}_{i j} \sigma_{z}\right) \delta_{i j} \delta\left(\tau-\tau^{\prime}\right)
$$


We note that formal power series expansion of $\Psi$ will contain only even powers in $\Delta$ and it has the form

$$
\operatorname{Tr} \ln \left(1-\hat{\Delta} \hat{\mathcal{G}}_{0}\right)=-\sum_{k=1}^{\infty} \frac{\operatorname{Tr}\left(\hat{\Delta} \hat{\mathcal{G}}_{0}\right)^{2 k}}{2 k} .
$$

The explicit form of the expansion up to the 4th order is given below

$$
\begin{aligned}
\Psi^{(4)} & =\sum_{\boldsymbol{q}, n}\left[\frac{1}{|U|}+\chi\left(\boldsymbol{q}, \mathrm{i} \varepsilon_{n}\right)\right]\left|\Delta_{q}\left(\mathrm{i} \varepsilon_{n}\right)\right|^{2} \\
& +\frac{1}{2 N \beta} \sum_{\boldsymbol{p}_{1} \boldsymbol{p}_{2} \boldsymbol{p}_{3}, n_{1} n_{2} n_{3}} \Gamma^{+-+-}\left(\boldsymbol{p}_{1}, \boldsymbol{p}_{2}, \boldsymbol{p}_{3} ; n_{1}, n_{2}, n_{3}\right) \Delta_{\boldsymbol{p}_{1}}^{*}\left(\mathrm{i} \varepsilon_{n_{1}}\right) \Delta_{\boldsymbol{p}_{2}}^{*}\left(\mathrm{i} \varepsilon_{n_{2}}\right) \\
& \times \Delta_{\boldsymbol{p}_{3}}\left(\mathrm{i} \varepsilon_{n_{3}}\right) \Delta_{\boldsymbol{p}_{1}+\boldsymbol{p}_{2}-\boldsymbol{p}_{3}}\left(\mathrm{i} \varepsilon_{n_{1}}+\mathrm{i} \varepsilon_{n_{2}}-\mathrm{i} \varepsilon_{n_{3}}\right) .
\end{aligned}
$$

In the above equation

$$
\chi\left(\boldsymbol{q}, \mathrm{i} \varepsilon_{n}\right)=\frac{1}{N} \sum_{k} \frac{f\left(\varepsilon_{k}-\mu\right)-f\left(-\varepsilon_{k+q}+\mu\right)}{\varepsilon_{k}+\varepsilon_{k+q}-2 \mu-\mathrm{i} \varepsilon_{n}}
$$

is the free particle pairing susceptibility, $f(x)=[\exp (\beta x)+1]^{-1}$ is the Fermi distribution function, $\varepsilon_{n}=\frac{2 \pi}{\beta} n$ are the bosonic Matsubara frequencies. $\Delta_{\boldsymbol{k}}\left(\mathrm{i} \varepsilon_{n}\right)$ is the Fourier transform of the field $\Delta_{i}(\tau)$ and is given by

$$
\Delta_{q}\left(\mathrm{i} \varepsilon_{n}\right)=\frac{1}{\sqrt{\beta N}} \sum_{j} \int_{0}^{\beta} \mathrm{d} \tau \mathrm{e}^{\mathrm{i}\left(\varepsilon_{n} \tau-\boldsymbol{q} \cdot \boldsymbol{R}_{j}\right)} \Delta_{j}(\tau) .
$$

The quartic term is of the form

$$
\begin{aligned}
\Gamma^{+-+-} & \left(\boldsymbol{p}_{1}, \boldsymbol{p}_{2}, \boldsymbol{p}_{3} ; n_{1}, n_{2}, n_{3}\right)=\frac{1}{\beta N} \sum_{\boldsymbol{k}, m} \mathcal{G}_{0}^{+}\left(k, \mathrm{i} \omega_{m}\right) \mathcal{G}_{0}^{-}\left(k+p_{1}, \mathrm{i} \omega_{m}-\mathrm{i} \varepsilon_{n}\right) \\
& \times \mathcal{G}_{0}^{+}\left(k+p_{3}-p_{2}, \mathrm{i} \omega_{m}+\mathrm{i} \varepsilon_{n_{2}}-\mathrm{i} \varepsilon_{n_{3}}\right) \mathcal{G}_{0}^{-}\left(k+p_{3}, \mathrm{i} \omega_{m}-\mathrm{i} \varepsilon_{n_{3}}\right)
\end{aligned}
$$

where $\hat{\mathcal{G}}_{0}^{-1}=\mathrm{i} \omega_{n}-\left(\varepsilon_{k}-\mu\right) \sigma_{z}$ is the Fourier transform of the free fermion Green function and $\omega_{n}=\frac{2 \pi}{\beta}\left(n+\frac{1}{2}\right)$ are the fermionic Matsubara frequencies. If we neglect all the frequency dependence of $\Delta$ in the expansion (17) (the static approximation), then we have the following result for the quartic term (we also neglect the momentum dependence)

$$
\Gamma^{+-+-}(0,0,0)=\frac{b}{2}=\frac{1}{\beta N} \sum_{k, m}\left[\frac{1}{\left(\mathrm{i} \omega_{m}\right)^{2}-\bar{\varepsilon}_{k}^{2}}\right]^{2}, \quad \bar{\varepsilon}_{k}=\varepsilon_{k}-\mu,
$$

which is the standard expression for the quartic term in the Ginzburg-Landau theory. We note that the corresponding coefficients can be evaluated explicitly and basing on such expansion one can study the question of the crossover from the BCS pairing (when $\mu \approx E_{\mathrm{F}}$ ) to the bosonic limit of tightly bound pairs $(\mu \approx$ $-E_{\mathrm{b}} / 2$, where $E_{\mathrm{b}}$ is the binding energy for two fermions in an empty lattice). For completeness, we give below the time-independent Ginzburg-Landau functional 


$$
\bar{\Psi} / \beta=\sum_{q}\left(\bar{a}+c \frac{q^{2}}{2 m}\right)\left|\Delta_{q}\right|^{2}+\frac{b}{2} \sum_{k_{1}, k_{2}, k_{3}} \Delta_{k_{1}}^{*} \Delta_{k_{2}}^{*} \Delta_{k_{3}} \Delta_{k_{1}+k_{2}-k_{3}},
$$

where $\Delta_{q}=\Delta_{q}(0)$. The coefficients appropriate to a weak coupling superconductor are given by

$$
\bar{a}=N\left(E_{\mathrm{F}}\right) \ln \frac{T}{T_{\mathrm{C}}}, \quad c=\frac{7 \zeta(3) N\left(E_{\mathrm{F}}\right) E_{\mathrm{F}}}{12 \pi^{2}\left(k_{\mathrm{B}} T_{\mathrm{C}}\right)^{2}}, \quad b=\frac{7 \zeta(3) N\left(E_{\mathrm{F}}\right)}{8 \pi^{2}\left(k_{\mathrm{B}} T_{\mathrm{C}}\right)^{2}},
$$

where $N\left(E_{\mathrm{F}}\right)$ is the density of states (DOS) at the Fermi level and $E_{\mathrm{F}}$ is the Fermi energy. The above G-L functional will be used in Sec. 6 in discussing the effects of amplitude fluctuations above $T_{c}$.

\section{From BCS to Bose superconductivity}

As mentioned above, given the functional integral representation (15), the evolution from BCS to Bose type superconductivity can be analyzed. The coefficient in quadratic term of (18) is of the form

$$
A\left(\boldsymbol{q}, \mathrm{i} \varepsilon_{n}\right)=\frac{1}{|U|}+\chi\left(\boldsymbol{q}, \mathrm{i} \varepsilon_{n}\right)
$$

It is convenient to eliminate the pairing potential $|U|$ in favor of the two-particle binding energy in vacuum, $E_{\mathrm{b}}[7]$, through the relation

$$
\frac{1}{|U|}=\frac{1}{N} \sum_{k} \frac{1}{2 \varepsilon_{k}+E_{\mathrm{b}}} .
$$

In the long-wave limit, assuming the parabolic spectrum for fermions with the mass $m$, the expansion is given by

$$
A\left(\boldsymbol{q}, \mathrm{i} \varepsilon_{n}\right)=a+c \frac{q^{2}}{2 m}-\mathrm{i} \bar{d} \varepsilon_{n}
$$

where

$$
\begin{aligned}
& \bar{a}=\frac{1}{N} \sum_{k} \frac{1}{2 \varepsilon_{k}+E_{\mathrm{b}}}-\frac{1}{N} \sum_{k} \frac{\tanh \left(\beta \bar{\varepsilon}_{k} / 2\right)}{2 \bar{\varepsilon}_{k}}, \\
& c=\frac{1}{N} \sum_{k}\left[\left(\frac{\tanh \left(\beta \bar{\varepsilon}_{k} / 2\right)}{4 \bar{\varepsilon}_{k}^{2}}-\frac{\beta}{8 \bar{\varepsilon}_{k} \cosh ^{2}\left(\beta \bar{\varepsilon}_{k} / 2\right)}\right)\right. \\
& \left.+\left(\frac{\beta^{2} \tanh \left(\beta \bar{\varepsilon}_{k} / 2\right)}{\cosh ^{2}\left(\beta \bar{\varepsilon}_{k} / 2\right)}+\frac{\beta}{\bar{\varepsilon}_{k} \cosh ^{2}\left(\beta \bar{\varepsilon}_{k} / 2\right)}-\frac{2 \tanh \left(\beta \bar{\varepsilon}_{k} / 2\right)}{\bar{\varepsilon}_{k}^{2}}\right) \frac{(k \cdot n)^{2}}{8 m \bar{\varepsilon}_{k}}\right], \\
& b / 2=\frac{1}{N} \sum_{k}\left(\frac{\tanh \left(\beta \bar{\varepsilon}_{k} / 2\right)}{4 \bar{\varepsilon}_{k}^{3}}-\frac{\beta}{8 \bar{\varepsilon}_{k}^{2}} \frac{1}{\cosh ^{2}\left(\beta \bar{\varepsilon}_{k} / 2\right)}\right),
\end{aligned}
$$

and the coefficient $\bar{d}$ will be specified later. 
In the $\mathrm{BCS} /$ Fermi liquid regime $E_{\mathrm{b}} / E_{\mathrm{F}} \ll 1, \mu \approx E_{\mathrm{F}} \gg T$ and we recover the standard form of the Ginzburg-Landau functional

$$
S_{\mathrm{GL}}=\sum_{q}\left(\bar{a}+c \frac{q^{2}}{2 m}-\mathrm{i} \bar{d} \varepsilon_{n}\right)\left|\Delta_{q}\right|^{2}+\frac{b}{2} \sum_{1,2,3} \Delta_{1}^{*} \Delta_{2}^{*} \Delta_{3} \Delta_{1+2-3}
$$

$q=\left(\boldsymbol{q}, \mathrm{i} \varepsilon_{n}\right)$, with the coefficients $\bar{a}, b, c$ given by Eqs. (24) and with $\bar{d}=$ $\pi N\left(E_{\mathrm{F}}\right) / 8 k_{\mathrm{B}} T_{\mathrm{c}}$. Indeed, with the rescaling $\Phi(k)=\sqrt{2 c} \Delta_{k}$ (the Gorkov relation), it takes the conventional form of the time-dependent $\mathrm{G}-\mathrm{L}$ functional.

In the opposite Bose limit $E_{\mathrm{b}} / E_{\mathrm{F}} \gg 1$, and the chemical potential $-\mu$ approaches $E_{\mathrm{b}} / 2$ at the transition point, thus $-\mu \gg T_{\mathrm{c}}$. Defining the effective chemical potential $\mu=\bar{\mu}-\frac{E_{\mathrm{b}}}{2}$, we then obtain $\bar{a}=-\frac{N(0)}{2 E_{\mathrm{b}}} 2 \bar{\mu}, c=\frac{N(0)}{4 E_{\mathrm{b}}}, \bar{d}=\frac{N(0)}{2 E_{\mathrm{b}}}$, where $N(0)$ is the density of states (DOS) in 2D. After the rescaling of the pair-field amplitude $\Phi\left(\boldsymbol{q}, \mathrm{i} \varepsilon_{n}\right)=\sqrt{\frac{N(0)}{2 E_{\mathrm{b}}}} \Delta_{q}\left(\mathrm{i} \varepsilon_{n}\right)$, the effective action becomes

$$
\begin{aligned}
S_{\mathrm{eff}} & =\sum_{q}\left(\frac{q^{2}}{2 m^{*}}-2 \bar{\mu}-\mathrm{i} \varepsilon_{n}\right)|\Phi(q)|^{2} \\
& +\bar{b} \sum_{1,2,3} \Phi^{*}(1) \Phi^{*}(2) \Phi(3) \Phi(1+2-3),
\end{aligned}
$$

where $m^{*}=2 m, \bar{\mu}=E_{\mathrm{b}} / 2+\mu$, and $\bar{b}$ is the parameter describing the interaction between the tightly bound pairs. Equation (32) yields required Gross-Pitaevski functional describing interacting bosons in the dilute limit, with the effective mass $m^{*}=2 m$ and the effective chemical potential $\bar{\mu}[20]^{*}$. The parameter $\bar{b}$ describes repulsive interaction between the bosons and it strongly depends on dimensionality. For a constant DOS, the above procedure yields

$$
b / 2=\frac{N(0)}{16} \frac{1}{|\mu|^{2}}
$$

where $N(0)=m / 2 \pi$. In the limit $|\mu| \rightarrow E_{\mathrm{b}} / 2$, the rescaling gives $\bar{b} \approx 2 / N(0)$. Thus, the only interaction is due to the Pauli principle for constituent fermions. However, in general, one would expect that the effective boson-boson repulsion should also be controlled by the scattering length for two bosons. In the strictly $d=2$ case, the scattering amplitude at long-waves and low-frequencies vanishes, so the above mapping must be taken with care, and the effects of higher orders in the expansion (18) may be of importance. We also notice that in $2 \mathrm{D}$, the coefficient $b$ of the GL functional (Eq. (33)), when $\mu \rightarrow 0$, is divergent, thus indicating a singular point in the evolution from BCS to Bose limit. This however can be again cured by taking into account the interaction between the fluctuations [22].

*For a recent analysis of the mapping of fermionic system with attraction to the composite boson limit see [21]. 
A question of the derivation of the time-dependent Ginzburg-Landau functional by this method, in the weak and strong coupling regimes, is discussed in Ref. [12].

Finally, we should mention that the effective mass of the bosons $m^{*}$ in the continuum limit approaches $2 \mathrm{~m}$. If the tight binding spectrum of the form $\varepsilon_{k}=$ $-2 t\left[\cos \left(k_{x} a\right)+\cos \left(k_{y} a\right)+\cos \left(k_{z} a\right)\right]$, a being the lattice constant, is used, one obtains the following expansion for $A\left(\boldsymbol{q}, \mathbf{i} \varepsilon_{n}\right)$ :

$$
A\left(\boldsymbol{q}, \mathrm{i} \varepsilon_{n}\right)=\bar{a}+C \boldsymbol{q}^{2}-\mathrm{i} d \varepsilon_{n}
$$

where $\bar{a}$ and $b$ are given by Eq. (28) and Eq. (31) and

$$
\begin{aligned}
C= & \frac{1}{2 N} \sum_{k}\left(\frac{f^{\prime}\left(\bar{\varepsilon}_{k}\right)}{2 \bar{\varepsilon}_{k}}+\frac{1-2 f\left(\bar{\varepsilon}_{k}\right)}{4 \bar{\varepsilon}_{k}^{2}}\right) 2 t a \cos \left(k_{x} a\right) \\
& +\frac{1}{2 N} \sum_{k}\left(\frac{f^{\prime \prime}\left(\bar{\varepsilon}_{k}\right)}{2 \bar{\varepsilon}_{k}}-\frac{2 f^{\prime}\left(\bar{\varepsilon}_{k}\right)}{4 \bar{\varepsilon}_{k}^{2}}-\frac{1-2 f\left(\bar{\varepsilon}_{k}\right)}{4 \bar{\varepsilon}_{k}^{3}}\right) 4 t^{2} a^{2} \sin ^{2}\left(k_{x} a\right),
\end{aligned}
$$

$f^{n}$ are the derivatives of the Fermi function. The coefficient $\bar{d}$ can be obtained by first making an analytical continuation $\chi\left(\boldsymbol{q}=\mathbf{0}, \mathbf{i} \varepsilon_{n}\right) \rightarrow \chi\left(\boldsymbol{q}=0, \omega+i 0^{+}\right)$and then expanding in $\omega[12]$. One gets

$$
\bar{d}=\frac{1}{N} \sum_{k} \frac{1-2 f\left(\bar{\varepsilon}_{k}\right)}{4 \bar{\varepsilon}_{k}^{2}}+\mathrm{i} \frac{\pi N(\mu) \beta}{8} \Theta(\mu),
$$

where $\Theta(\mu)$ is the Heaviside step function. In the Bose limit the coefficients take the form: $\bar{a}=-2 \bar{\mu} / U^{2}, C=2 t^{2} a^{2} /|U|^{3}, d=1 / U^{2}$, and upon rescaling the pair-field amplitudes one gets the functional (33), with the bosonic mass $m^{*}=|U| /\left(4 t^{2} a^{2}\right)$. This result is in accord with the perturbation expansion for the attractive Hubbard model in the strong coupling limit [1].

\subsection{Quasi-two-dimensional case}

Let us now consider the quasi-two-dimensional case and assume the following spectrum for the fermions interacting via the on-site attractive potential $U$ :

$$
\varepsilon_{k}=\frac{k^{2}}{2 m_{\|}}-2 t_{\perp} \cos \left(k_{z} d\right)
$$

where $k^{2}=k_{x}^{2}+k_{y}^{2}, m_{\|}$is the in-plane fermion mass and $t_{\perp}$ is the hopping amplitude between the neighboring planes at distance $d$. In contrast to the strictly $2 \mathrm{D}$ case, now the $s$-wave BCS pairing and the bound state formation are not necessarily related to each other. The bound state condition, Eq. (26), shows that for a given ratio of $t_{\|} / t_{\perp}$ the threshold must be reached for $|U|$, to form the bound state in vacuum. In the BCS case, evaluation of the quadratic and quartic coefficients will yield an appropriate generalization of the $\mathrm{G}-\mathrm{L}$ functional to the quasi-2D case. 
Let us consider the strong coupling case above the threshold to form the bound pair, and where $\mu$ is below the bottom of the fermionic band and the temperature region $k_{\mathrm{B}} T<E_{\mathrm{b}}$. In such a case we can neglect the Fermi factors in the evaluation of the quadratic coefficient and obtain

$$
\begin{aligned}
A\left(\boldsymbol{q}, \mathrm{i} \varepsilon_{n}\right) & =\frac{1}{N} \sum_{k} \frac{1}{\frac{k^{2}}{m_{\|}}-4 t_{\perp} \cos \left(k_{z} d\right)+4 t_{\perp}+E_{\mathrm{b}}} \\
& -\frac{1}{N} \sum_{k} \frac{1}{\frac{k^{2}}{m_{\|}}+\frac{q^{2}}{4 m_{\|}}-4 t_{\perp} \cos \left(q_{z} d / 2\right) \cos \left(k_{z} d\right)+4 t_{\perp}-2 \mu-\mathrm{i} \varepsilon_{n}},
\end{aligned}
$$

and $q=\left(q, q_{z}\right)$.

After integrating over $k$, one gets

$$
\begin{aligned}
A\left(\boldsymbol{q}, \mathrm{i} \varepsilon_{n}\right) & =\frac{N(0)}{2} \\
& \times \ln \left(\frac{\frac{q^{2}}{4 m_{\|}}-2 \mu-\mathrm{i} \varepsilon_{n}+\sqrt{\left(\frac{q^{2}}{4 m_{\|}}-2 \mu-\mathrm{i} \varepsilon_{n}\right)^{2}-16 t_{\perp}^{2} \cos ^{2}\left(\frac{q_{z} d}{2}\right)}}{E_{\mathrm{b}}+4 t_{\perp}+\sqrt{\left(E_{\mathrm{b}}+4 t_{\perp}\right)^{2}-16 t_{\perp}^{2}}}\right) .
\end{aligned}
$$

Introducing the effective chemical potential by the relation $\mu=-\frac{1}{2} E_{\mathrm{b}}-$ $2 t_{\perp}+\bar{\mu}$, and expanding (39) to lowest order in $q_{\|}$and $i \varepsilon_{n}$, we obtain

$$
A\left(\boldsymbol{q}, \mathrm{i} \varepsilon_{n}\right)=\frac{N(0)}{2 E_{\mathrm{b}}}\left(\frac{q^{2}}{4 m_{\|}}+\frac{\left(4 t_{\perp}\right)^{2}}{8 E_{\mathrm{b}}}\left[1-\cos \left(q_{z} d\right)\right]-2 \bar{\mu}-\mathrm{i} \varepsilon_{n}\right) .
$$

By rescaling the pairing-field amplitude $\Phi\left(\boldsymbol{q}, \mathrm{i} \varepsilon_{n}\right)=\sqrt{\frac{N(0)}{2 E_{\mathrm{b}}}} \Delta_{q}\left(\mathrm{i} \varepsilon_{n}\right)$, one obtains the Gross-Pitaevski action for the quasi-2D-bosons

$$
\begin{aligned}
S_{\mathrm{eff}} & =\sum_{q}\left(\frac{q^{2}}{4 m_{\|}}+\frac{1}{2 m_{\perp}^{2} d^{4} E_{\mathrm{b}}}\left[1-\cos \left(q_{z} d\right)\right]-2 \bar{\mu}-\mathrm{i} \varepsilon_{n}\right)|\Phi(q)|^{2} \\
& +\tilde{b} \sum_{1,2,3} \Phi^{*}(1) \Phi^{*}(2) \Phi(3) \Phi(1+2-3),
\end{aligned}
$$

where $m_{\perp}=1 / t_{\perp}^{2} d^{2}$ is the fermionic mass in the perpendicular direction. The bosons have the in-plane mass $2 m_{\|}$and the perpendicular mass $m_{\perp}^{*}=2 E_{\mathrm{b}} m_{\perp}^{2} d^{2}$ being much greater than $m_{\perp}$. The physical origin of the increased perpendicular mass is clear, to move the pair between the planes one has to break it first. Indeed, these (composite) bosons will condense at the temperature given by [23]

$$
k_{\mathrm{B}} T_{\mathrm{c}}=\frac{\pi n^{*} d}{2 m_{\|} \ln \left(2 k_{\mathrm{b}} T_{\mathrm{c}} \nu E_{\mathrm{b}} m_{\|}^{2} d^{4}\right)} \approx \frac{E_{\mathrm{F}}}{2 \ln \left(4 k_{\mathrm{b}} T_{\mathrm{c}} E_{\mathrm{b}} m_{\|}^{2} d^{4}\right)},
$$


$n^{*}=n / 2$ is the boson density, $n$ being the electron density and $E_{\mathrm{F}}=\pi n d / m_{\|}$. Thus, the transition temperature for the quasi-two-dimensional case in the preformed pair regime is proportional to $E_{\mathrm{F}}$. This temperature will decrease with increasing interaction making the pair binding stronger. We should point out that independently, the same result has been obtained in Ref. [24], by using a different approach.

\subsection{The extended Hubbard model with pair-hopping interaction}

The above derivations of the Ginzburg-Landau functionals can be extended to the case of the Hubbard model with the pair hopping interaction (the PensonKolb-Hubbard model), which involves the non-local pairing interaction and is given by the Hamiltonian

$$
H=\sum_{i, j, \sigma} \hat{t}_{i j} c_{i \sigma}^{\dagger} c_{j \sigma}+U \sum_{i} \rho_{i}^{\dagger} \rho_{i}-\frac{1}{2} \sum_{i, j}\left(J_{i j} \rho_{i}^{\dagger} \rho_{j}+\text { H.c. }\right),
$$

where $J_{i j}$ is the pair-hopping (intersite charge exchange) parameter [25]. The functional integral representation of the partition function of the model (43) is given by Eq. (15) with

$$
\Psi=\sum_{\boldsymbol{q}, n} \frac{\left|\Delta_{q}\left(\mathrm{i} \varepsilon_{n}\right)\right|^{2}}{V_{q}}-\operatorname{Tr} \ln \left(1-\hat{\Delta} \hat{\mathcal{G}}_{0}\right),
$$

where $V_{\boldsymbol{q}}=-U+J_{\boldsymbol{q}}$ and $J_{\boldsymbol{q}}=2 J\left[\cos \left(k_{x} a\right)+\cos \left(k_{y} a\right)+\cos \left(k_{z} a\right)\right]$ is the Fourier transform of $J_{i j}$. The coefficient of the quadratic term in the power series expansion of $\Psi$ in $\Delta$ is

$$
A\left(\boldsymbol{q}, \mathrm{i} \varepsilon_{n}\right)=\frac{1}{V_{\boldsymbol{q}}}+\chi\left(\boldsymbol{q}, \mathrm{i} \varepsilon_{n}\right),
$$

where the pairing susceptibility is determined from Eq. (19). The small $\boldsymbol{q}, \omega$ expansion is now of the following form:

$$
A\left(\boldsymbol{q}, \mathrm{i} \varepsilon_{n}\right)=\bar{a}_{1}+C_{1} \boldsymbol{q}^{2}-\mathrm{i} d \varepsilon_{n},
$$

where

$$
\begin{aligned}
& \bar{a}_{1}=\frac{1}{V_{0}}-\frac{1}{N} \sum_{k} \frac{\tanh \left(\beta \bar{\varepsilon}_{k} / 2\right)}{2 \bar{\varepsilon}_{k}}=\frac{1}{N} \sum_{k} \frac{1}{2 \varepsilon_{k}+E_{\mathrm{b}}}-\frac{1}{N} \sum_{k} \frac{\tanh \left(\beta \bar{\varepsilon}_{k} / 2\right)}{2 \bar{\varepsilon}_{k}}, \\
& C_{1}=\frac{J a^{2}}{V_{0}^{2}}+C,
\end{aligned}
$$

and $C$ is given by Eq. (35). The complete expansion of the functional (44) to the 4 th order is fully specified by the above expressions together with the coefficient $\bar{d}$ given by Eq. (36) and $b$ given by Eq. (30). 


\section{Gaussian fluctuations and the T-matrix approach}

In this section we will analyze the contribution from the Gaussian fluctuations to the free energy and the self-consistent T-matrix approach. The effect of Gaussian fluctuations of the order parameter is obtained by keeping the quadratic terms only in the full functional. The corresponding contribution to the thermodynamic potential is simply given by

$$
\beta \mathcal{F}^{(2)}=\frac{1}{N} \sum_{\boldsymbol{q}, n} \ln \left[1+|U| \chi\left(\boldsymbol{q}, \mathrm{i} \varepsilon_{n}\right)\right]
$$

where

$$
\chi\left(\boldsymbol{q}, \mathrm{i} \varepsilon_{n}\right)=\frac{-1}{\beta N} \sum_{\boldsymbol{k}, m} G_{0}\left(\boldsymbol{k}, \mathrm{i} \omega_{m}\right) G_{0}\left(\boldsymbol{q}-\boldsymbol{k}, \mathrm{i} \varepsilon_{n}-\mathrm{i} \omega_{m}\right),
$$

with $G_{0}\left(\boldsymbol{k}, \mathrm{i} \omega_{n}\right)^{-1}=\mathrm{i} \omega_{n}-\left(\varepsilon_{k}-\mu\right)$, and is given by Eq. (19).

The BCS pairing instability corresponds to a singularity for $\boldsymbol{q}=\mathbf{0}, \boldsymbol{\omega}=0$ in $\mathcal{F}^{(2)}$, and the condition (the Thouless criterion) $1+|U| \chi(0,0)=0$ gives $T_{\mathrm{c}}$.

Equations (48), (49) can be used to form a fully renormalized, conserving approximation for the free energy and Green function [26-28]. Let the functional $\Phi[G]$ be defined as

$$
\begin{aligned}
& \Phi[G]=\frac{1}{\beta N} \sum_{\boldsymbol{q}, n} \ln \left[1-|U| \bar{\chi}\left(\boldsymbol{q}, \mathrm{i} \varepsilon_{n}\right)\right], \\
& \bar{\chi}\left(\boldsymbol{q}, \mathrm{i} \varepsilon_{n}\right)=\frac{1}{\beta N} \sum_{\boldsymbol{k}, m} G\left(\boldsymbol{k}, \mathrm{i} \omega_{m}\right) G\left(\boldsymbol{q}-\boldsymbol{k}, \mathrm{i} \varepsilon_{n}-i \omega_{m}\right),
\end{aligned}
$$

where $G\left(\boldsymbol{k}, \mathrm{i} \omega_{n}\right)$ is the full Green function and we use conventional definition of the pairing susceptibility Eq. (51).

The corresponding self-consistent approximation for the free energy is given by the functional

$$
\begin{aligned}
& \mathcal{F}[\Sigma, G]=-\frac{2}{\beta N} \sum_{\boldsymbol{k}, n} \exp \left(\mathrm{i} \omega_{n} \eta\right)\left\{\Sigma\left(\boldsymbol{k}, \mathrm{i} \omega_{n}\right) G\left(\boldsymbol{k}, \mathrm{i} \omega_{n}\right)+\ln \left[-G_{0}\left(\boldsymbol{k}, \mathrm{i} \omega_{n}\right)^{-1}\right.\right. \\
& \left.\left.\quad+\Sigma\left(\boldsymbol{k}, \mathrm{i} \omega_{n}\right)\right]\right\}+\Phi[G]
\end{aligned}
$$

evaluated at its stationary point with respect to variations of $G$ and $\Sigma$. At this stationary point $G, \Sigma$, and $\Phi$ are related by

$$
\begin{aligned}
& G\left(\boldsymbol{k}, \mathrm{i} \omega_{n}\right)=\left[G_{0}\left(\boldsymbol{k}, \mathrm{i} \omega_{n}\right)^{-1}-\Sigma\left(k, \mathrm{i} \omega_{n}\right)\right]^{-1}, \\
& \Sigma\left(\boldsymbol{k}, \mathrm{i} \omega_{n}\right)=\frac{1}{2} \frac{\delta \Phi[G]}{\delta G\left(\boldsymbol{k}, \mathrm{i} \omega_{n}\right)} .
\end{aligned}
$$

Evaluation of $\Sigma\left(\boldsymbol{k}, \mathrm{i} \omega_{n}\right)$ yields 


$$
\Sigma\left(\boldsymbol{k}, \mathrm{i} \omega_{n}\right)=\frac{1}{\beta N} \sum_{\boldsymbol{q}, m} T\left(\boldsymbol{q}, \mathrm{i} \varepsilon_{m}\right) G\left(\boldsymbol{q}-\boldsymbol{k}, \mathrm{i} \varepsilon_{m}-\mathrm{i} \omega_{n}\right)
$$

where

$$
T\left(\boldsymbol{q}, \mathrm{i} \varepsilon_{n}\right)=\frac{-|U|}{1-|U| \bar{\chi}\left(\boldsymbol{q}, \mathrm{i} \varepsilon_{n}\right)}
$$

is the T-matrix.

The electron concentration as a function of $T$ and $\mu$ is obtained as $n(T, \mu)=$ $-\partial \mathcal{F} / \partial \mu$. By using stationary properties of $\mathcal{F}$ one gets the expected result

$$
n(T, \mu)=\frac{2}{\beta N} \sum_{\boldsymbol{k}, n} \exp \left(\mathrm{i} \omega_{n} \eta\right) G\left(\boldsymbol{k}, \mathrm{i} \omega_{n}\right) .
$$

The above equations (53), (55), (56), (51), (57) constitute the system of self-consistent $\mathbf{T}$-matrix equations and they were recently analyzed numerically for the attractive Hubbard model in two dimensions (mostly in low concentration regime) [29].

\section{Self-consistent approach to the superconducting fluctuations}

\subsection{Hartree theory and variational principle approach}

We would like to derive the best Gaussian form for the superconducting fluctuations. Let us seek for a trial functional $\Psi_{\mathrm{T}}$ in the following form:

$$
\Psi_{\mathrm{T}}\left[\Delta_{q}\right]=\sum_{q} A_{q}\left|\Delta_{q}\right|^{2}
$$

where $q=\left(q, \mathrm{i} \varepsilon_{n}\right)$. Application of the Feynman variational principle allows us to write the corresponding variational free energy $F_{0}$ :

$$
\beta F_{0}=-\ln \int D^{2} \Delta \mathrm{e}^{-\Psi_{\mathrm{T}}\left[\Delta_{q}\right]}+\left\langle\Psi-\Psi_{\mathrm{T}}\right\rangle_{\mathrm{T}},
$$

where $\langle\cdots\rangle_{\mathrm{T}}=\int D^{2} \Delta \exp \left(-\Psi_{\mathrm{T}}\left[\Delta_{q}\right]\right)(\cdots) / \int D^{2} \Delta \exp \left(-\Psi_{\mathrm{T}}\left[\Delta_{q}\right]\right)$. To evaluate the right hand side (RHS) of Eq. (59) we consider two cases:

1. We use expansion of the exact functional $\Psi$ truncated at the 4 th order term.

2. We use the exact form of $\Psi$ given by Eq. (15).

In the first case we use $\Psi$ as given by Eq. (18) and after exploring the properties of Gaussian integrals we obtain for $F_{0}$

$$
\begin{aligned}
\beta F_{0} & =-\ln \int D^{2} \Delta^{-\Psi_{\mathrm{T}}\left[\Delta_{q}\right]}+\sum_{q}\left[\frac{1}{|U|}+\chi(q)-A_{q}\right]\left\langle\left|\Delta_{q}\right|^{2}\right\rangle \\
& +\frac{1}{2 N \beta} \sum_{p_{1}, p_{2}} \Gamma^{+-+-}\left(p_{1}, p_{2}, p_{2}\right)\left\langle\left|\Delta_{p_{1}}\right|^{2}\right\rangle\left\langle\left|\Delta_{p_{2}}\right|^{2}\right\rangle
\end{aligned}
$$




$$
+\frac{1}{2 N \beta} \sum_{p_{1}, p_{2}} \Gamma^{+-+-}\left(p_{1}, p_{2}, p_{1}\right)\left\langle\left|\Delta_{p_{1}}\right|^{2}\right\rangle\left\langle\left|\Delta_{p_{2}}\right|^{2}\right\rangle
$$

where $\left\langle\left|\Delta_{p}\right|^{2}\right\rangle$ is the functional average with $\Psi_{\mathrm{T}}$. The best coefficients $A_{q}$ are obtained from the stationary condition $\delta\left(\beta F_{0}\right) / \delta A_{q}=0$. With the notation $t_{q}=$ $\left\langle\left|\Delta_{q}\right|^{2}\right\rangle$ for the mean square amplitude of the order parameter (fluctuating in space and time), we obtain

$$
A_{q}=\frac{1}{|U|}+\chi(q)+\frac{1}{2 N \beta} \sum_{p}\left[\Gamma^{+-+-}(q, p, p)+\Gamma^{+-+-}(p, q, p)\right] t_{p} .
$$

Since $\Psi_{\mathrm{T}}$ is the Gaussian functional then $t_{\mathrm{q}}=1 / A_{q}$, and we obtain the following self-consistent equation for $t_{q}$ :

$$
t_{q}=\left\{\frac{1}{|U|}+\chi(q)+\frac{1}{2 N \beta} \sum_{p}\left[\Gamma^{+-+-}(q, p, p)+\Gamma^{+-+-}(p, q, p)\right] t_{p}\right\}^{-1} .
$$

If we neglect the frequency dependence and perform small momentum expansion for $\chi(q)$ and also neglect the momentum dependence of the $\Gamma$ terms in (62), then we recover the Hartree equation for the amplitude fluctuations used in Sec. 6.

\subsection{Fully self-consistent approach}

In the second case we use the complete functional (15) to evaluate the trial free energy $(59)^{\dagger}$. By making the substitution $\Delta_{q}=A_{q}^{-1 / 2} \xi_{q}$, one gets

$$
\beta F_{0}=-\sum_{q}\left[1-\ln \left(|U| A_{q}\right)\right]+\int D^{2} \xi_{q} \mathrm{e}^{-\sum_{q}\left|\xi_{q}\right|^{2}} \Psi\left[A_{q}^{-1 / 2} \xi_{q}\right] .
$$

The stationary condition $\delta\left(\beta F_{0}\right) / \delta A_{q}=0$, after integrating by parts, yields the following expression for $A_{q}$ :

$$
A_{q}=\left\langle\frac{\delta^{2} \Psi}{\delta \Delta_{q} \delta \Delta_{q}^{*}}\right\rangle_{\mathrm{T}}
$$

i.e. the optimal quadratic functional coefficients are self-consistently averaged second derivatives of the true functional. We note that the random phase approximation (RPA) result is obtained if the second derivative is evaluated at the origin

$$
A_{q}=\left.\frac{\delta^{2} \Psi}{\delta \Delta_{q} \delta \Delta_{q}^{*}}\right|_{\Delta=0}
$$

and it simply yields Eq. (25) with the free particle-particle susceptibility (19). By using Eq. (64) and the free energy functional Eq. (15), after tedious manipulations (similar to those in Ref. [31]), one arrives at

$$
\frac{\delta^{2}}{\delta \Delta_{q} \delta \Delta_{q}^{*}}\left(-\operatorname{Tr} \ln \left(1-\hat{\Delta} \hat{\mathcal{G}}_{0}\right)\right)=\sum_{k, k^{\prime}} \mathcal{G}_{k, k^{\prime}}^{11}\{\Delta\} \mathcal{G}_{k+q, k^{\prime}+q}^{22}\{\Delta\}
$$

\footnotetext{
${ }^{\dagger}$ For an early study of variational treatment see [30].
} 
where $\mathcal{G}_{k k^{\prime}}^{\alpha \alpha}\{\Delta\}, \alpha=1,2$, denotes the diagonal matrix element of the space-time Fourier transform of $\hat{\mathcal{G}}_{i, j}\left(\tau, \tau^{\prime},\{\Delta\}\right)$ given by Eq. (14). We note that $\hat{\mathcal{G}}\{\Delta\}=$ $\hat{\mathcal{G}}_{0}\left(1-\hat{\Delta} \hat{\mathcal{G}}_{0}\right)^{-1}$ is the electron propagator in the random potential $\Delta_{i}(\tau)$. The expression for thecoefficient $A_{q}=A\left(\boldsymbol{q}, \mathrm{i} \epsilon_{n}\right)$ is then given by

$$
A\left(\boldsymbol{q}, \mathrm{i} \epsilon_{n}\right)=\frac{1}{|U|}+\bar{\chi}\left(\boldsymbol{q}, \mathrm{i} \epsilon_{n}\right)
$$

where

$\bar{\chi}\left(\boldsymbol{q}, \mathrm{i} \epsilon_{n}\right)=\frac{1}{\beta N} \sum_{\boldsymbol{k}, \boldsymbol{k}^{\prime}, m}\left\langle\mathcal{G}^{11}\left(\boldsymbol{k}, \boldsymbol{k}^{\prime}, \mathrm{i} \omega_{m} ;\{\Delta\}\right) \mathcal{G}^{22}\left(\boldsymbol{k}+\boldsymbol{q}, \boldsymbol{k}^{\prime}+\boldsymbol{q}, \mathrm{i} \omega_{m+n} ;\{\Delta\}\right)\right\rangle_{\mathrm{T}}$.

Therefore, we have derived a self-consistent theory of superconducting fluctuations in which the pairing susceptibility $\bar{\chi}\left(k, i \epsilon_{n}\right)$ is given by the averaged particleparticle propagator in the presence of the random fields. In this approach the particle-particle propagator in Eq. (67) is just the product of two local Green functions self-consistently averaged with the Gaussian weight $\exp \left(-A_{q}\left|\Delta_{q}\right|^{2}\right.$ ). (To lowest order in $|U|$, when we ignore the random fields, $\bar{\chi}$ reduces to the bare $\chi$, and we recover the RPA result.) The above theory is very similar in the spirit to the paramagnetic polaron theory in itinerant-electron magnetism [31] and can be used to describe incoherent local pair state. Finally, we note that the presented theory when resorting to the static approximation yields a theoretical foundation for the approach in which the electronic DOS is evaluated with the assumption of existence of the local order parameter amplitude above $T_{\mathrm{c}}$ and averaged with the Gaussian weight.

\section{Amplitude fluctuations above $T_{\mathrm{c}}$ in the Ginzburg-Landau theory and incoherent local pair state}

It is of interest to study further the fluctuation effects (and pairing correlations) and their role in establishing pair formation above $T_{c}$, within the conventional Ginzburg-Landau theory. From the previous analysis, one comes to the conclusion that above $T_{\mathrm{c}}$ there will be a region of parameters in which there are pairs with amplitude and phase fluctuating in space and time. Such a state, however, has no long-range phase coherence which is necessary to establish the superconducting state. This scenario is somewhat similar to a local-moment itinerant electron magnetism in transition metals and alloys, in which the magnetic moments exist above transition temperature but their direction is random. A possibility of such a state in the negative $-U$ Hubbard model has been considered earlier [1] to describe the intermediate region between BCS and the local pair regime. Such an incoherent local pair state has also been analyzed by Gyorffy et al. [32] and Park et al. [33] within the coherent potential approximation to the negative $-U$ Hubbard model with the use of a constant density of states. 
In order to show the existence of a finite gap amplitude above $T_{c}$, we consider the standard Ginzburg-Landau functional and apply the Hartree approximation to treat the interaction between superconducting fluctuations [33].

In zero magnetic field one has

$$
F[\Psi(\boldsymbol{r})]=\int \mathrm{d} \boldsymbol{r}\left[\bar{a}(t)|\Psi(\boldsymbol{r})|^{2}+\bar{b}|\Psi(\boldsymbol{r})|^{4}+\frac{\hbar^{2}}{4 m}|\nabla \Psi(\boldsymbol{r})|^{2}\right]
$$

where $t=T / T_{\mathrm{c}}$ and $m$ is an effective electron mass. The superconducting order parameter $\Psi(\boldsymbol{r})$ can be related to the gap parameter $\Delta(\boldsymbol{r})$, for example, via the Gorkov relation: $\Psi(r)=\left(\frac{7 \zeta(3) n}{8\left(\pi k_{\mathrm{B}} T_{\mathrm{c}}\right)^{2}}\right)^{1 / 2} \Delta(r)$, with $n$ the number of electrons per unit volume. With the Fourier transform to reciprocal space we have

$$
F\left[\Delta_{k}\right] / \Omega=\sum_{\boldsymbol{k}}\left\{\left[a(t)+c k^{2}\right]\left|\Delta_{k}\right|^{2}+b \sum_{\boldsymbol{p}, \boldsymbol{q}} \Delta_{k} \Delta_{\boldsymbol{p}}^{*} \Delta_{q} \Delta_{k-\boldsymbol{p}+\boldsymbol{q}}^{*}\right\},
$$

where $\Omega$ is the volume of the system. Assuming that $b$ and $c$ are temperature independent and performing the simplest quadratic factorization of the last term we obtain the Gaussian form of the $\mathrm{G}-\mathrm{L}$ functional

$$
F\left[\Delta_{k}\right] / \Omega=\sum_{\boldsymbol{k}}\left[a(t)+c k^{2}+2 b \sum_{q}\left\langle\left|\Delta_{q}\right|^{2}\right\rangle\right]\left|\Delta_{k}\right|^{2} .
$$

In the above equation the expectation value has to be calculated as the functional average

$$
\langle\ldots\rangle=\frac{\int D^{2} \Delta(\ldots) \exp \left[-F\left[\Delta_{k}\right] /\left(k_{\mathrm{B}} T\right)\right]}{\int D^{2} \Delta \exp \left[-F\left[\Delta_{k}\right] /\left(k_{\mathrm{B}} T\right)\right]} .
$$

The mean square gap amplitude $\Delta^{2}=\sum_{k}\left\langle\left|\Delta_{k}\right|^{2}\right\rangle$ is then given by the following self-consistent equation:

$$
\Delta^{2}=\frac{k_{\mathrm{B}} T}{\Omega} \sum_{k}\left[\frac{1}{a(t)+c k^{2}+2 b \Delta^{2}}\right] .
$$

In $2 \mathrm{D}$ we obtain

$$
\ln (1+w / x)=(\tilde{d} / t)(x+1-t)
$$

where the following relations have been used:

$$
\begin{aligned}
& a(t)=a(0)(1-t), \quad t=T / T_{\mathrm{c}}, \quad\left|\Delta_{0}\right|^{2}=-a(0) / 2 b, \quad x=\Delta^{2} /\left|\Delta_{0}\right|^{2}-1+t, \\
& w=\left(k_{\mathrm{c}} \xi_{0}\right)^{2}, \quad \tilde{d}=\frac{4 \pi|a(0)| \xi_{0}^{2}\left|\Delta_{0}\right|^{2}}{k_{\mathrm{B}} T_{\mathrm{c}}}, \quad a(0)=-\frac{7 \zeta(3) \hbar^{2} n\left|\Delta_{0}\right|^{2}}{8 \pi m\left(k_{\mathrm{B}} T_{\mathrm{c}}\right)^{2}} .
\end{aligned}
$$

In $3 \mathrm{D}$ case we get

$$
w^{*}-\sqrt{x} \arctan \left(\frac{w^{*}}{\sqrt{x}}\right)=\left(d^{*} / t\right)(x+1-t),
$$

where 


$$
w^{*}=k_{c} \xi_{0}, \quad d^{*}=\frac{7 \zeta(3) \hbar^{2} n\left|\Delta_{0}\right|^{2} \xi_{0}}{16 m\left(k_{\mathrm{B}} T_{\mathrm{C}}\right)^{3}}
$$

$\xi_{0}$ and $\Delta_{0}$ are the zero temperature coherence length and the zero temperature energy gap, respectively, $k_{\mathrm{c}}$ denotes the momentum cut-off.

We have solved these equations with the use of parameters appropriate for $\mathrm{YBa}_{2} \mathrm{Cu}_{3} \mathrm{O}_{7}$, taking $\left|\Delta_{0}\right|=20 \mathrm{meV}, T_{\mathrm{c}}=92 \mathrm{~K}, n=6 \times 10^{14} \mathrm{~cm}^{-2}$ (for $2 \mathrm{D}$ case) and $n \approx 10^{21} \mathrm{~cm}^{-3}$ (for $3 \mathrm{D}$ case), $m=5 m_{\mathrm{e}}, k_{\mathrm{c}}=\xi_{0}^{-1}$. The result is illustrated in Figs. 1 and 2 for several values of $\tilde{d}$ and $d^{*}$. For both $2 \mathrm{D}$ and $3 \mathrm{D}$ cases for $T$ above $T_{\mathrm{c}}, \Delta$ smoothly decreases and saturates at a finite value. Depending on the values of $\tilde{d}$ and $d^{*}, \Delta$ is of order $(0.1-0.2) \Delta_{0}$ for $2 \mathrm{D}$ case and of order $(0.05-0.125) \Delta_{0}$ in $3 \mathrm{D}$.
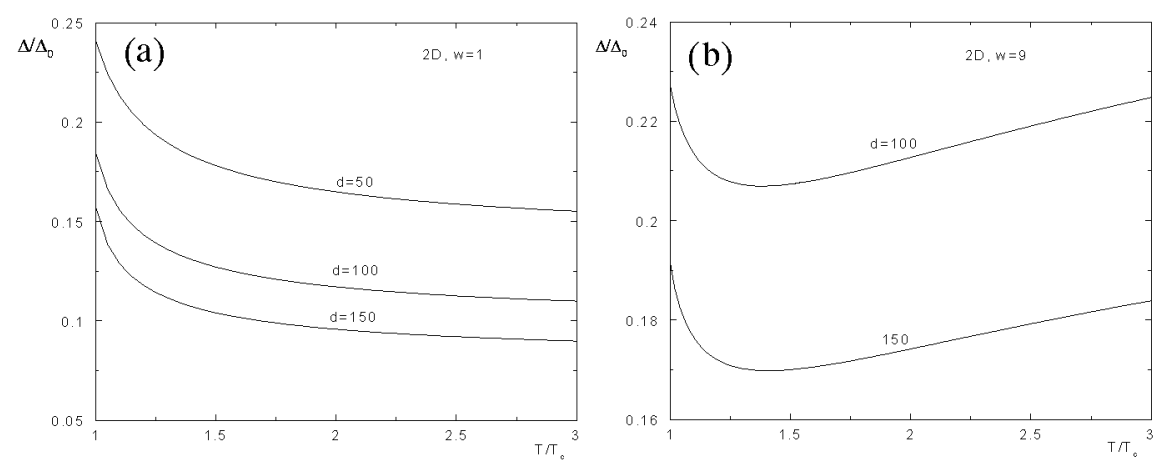

Fig. 1. $\Delta / \Delta_{0}$ versus $T / T_{\mathrm{c}}$ in two dimensions for two different values of the cut-off $k_{\mathrm{c}}$.
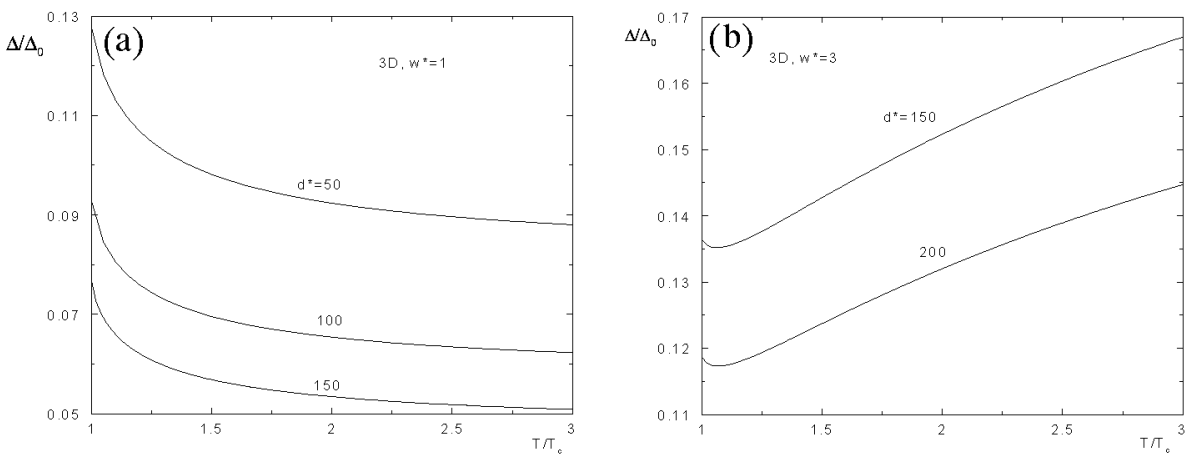

Fig. 2. $\Delta / \Delta_{0}$ versus $T / T_{\mathrm{c}}$ in three dimensions for two different values of the cut-off $k_{\mathrm{c}}$.

The parameter $k_{c}$ is not precisely defined in the Ginzburg-Landau theory and we consider it to vary. If we allow randomness of $\Delta(r)$ down to the atomic scale, then $k_{c}$ can be taken as an inverse of the lattice constant ( $4 \AA$ ), giving 
$w=9(2 \mathrm{D})$ and $w^{*}=3(3 \mathrm{D})$. In this case, we observe (Figs. 1 and 2 ) that after an initial decrease, $\Delta / \Delta_{0}$ starts to increase and saturates at larger values. The gap magnitude is of the order $(0.15-0.275) \Delta_{0}$ in $2 \mathrm{D}$ and $(0.1-0.2) \Delta_{0}$ in $3 \mathrm{D}$, depending on the values of the parameters $\tilde{d}$ and $d^{*}$. Thus if we lower $k_{\mathrm{c}}$, increasing temperature is more effective in making larger thermal fluctuations in the amplitude of the local order parameter $\Delta(r)$. This characteristic behavior is very similar to that known in the theory of local moment magnetism [34]. The above calculations carried out at the phenomenological Ginzburg-Landau level clearly illustrate the importance of the fluctuations above $T_{\mathrm{c}}$ and point out toward a possibility of existence of a state with incoherent pairs. In the incoherent local pair state the one-particle density of states will develop a pseudogap or real gap depending on the electron density and the strength of the attractive interaction.

\section{Acknowledgments}

A part of this work has been initiated at IBM Research Division-Rüschlikon, Switzerland. Thanks are due to H. Beck, S. Robaszkiewicz, and T. Schneider for helpful discussions.

\section{References}

[1] R. Micnas, J. Ranninger, S. Robaszkiewicz, Rev. Mod. Phys. 62, 113 (1990).

[2] Y.J. Uemura, L.P. Le, G.M. Luke, B.J. Sternlieb, W.D. Wu, J.H. Brewer, T.M. Riseman, C.L. Seaman, M.B. Maple, M. Ishikawa, D.G. Hinks, J.D. Jorgensen, G. Saito, H. Yamochi, Phys. Rev. Lett. 66, 2665 (1991); Y.J. Uemura, A. Keren, L.P. Le, G.M. Luke, B.J. Sternlieb, W.D. Wu, J.H. Brewer, R.L. Whetten, S.M. Huang, S. Lin, R.B. Kaner, F. Diederich, S. Donovan, G. Gruner, K. Holczer, Nature 352, 605 (1991)

[3] T. Schneider, H. Keller, Phys. Rev. Lett. 69, 3374 (1992); T. Schneider, H. Keller, Int. J. Mod. Phys. B 8, 487 (1994).

[4] R. Micnas, S. Robaszkiewicz, in: High- $T_{\mathrm{c}}$ Superconductivity 1996: Ten Years after the Discovery, Eds. E. Kaldis, E. Liaropkapis, K.A. Müller, NATO ASI Series E, Vol. 343, Kluwer 1997 , p. 31 and refs. therein.

[5] J. Orenstein, A.J. Millis, Science 288, 468 (2000).

[6] D.M. Eagles, Phys. Rev. 186, 456 (1969).

[7] A.J. Leggett, in: Modern Trends in the Theory of Condensed Matter, Eds. A. Pękalski, J. Przystawa, Springer Verlag, Berlin 1980, p. 13.

[8] S. Robaszkiewicz, R. Micnas, K.A. Chao, Phys. Rev. B 23, 1447 (1981); ibid. B 24, 1579 (1981); B 24, 4018 (1981); B 26, 3915 (1982).

[9] P. Nozieres, S. Schmitt-Rink, J. Low Temp. Phys. 59, 195 (1985).

[10] K. Miyake, Prog. Theor. Phys. 69, 195 (1983).

[11] M. Randeria, J.-M. Duan, L.-Y. Shieh, Phys. Rev. Lett. 62, 981 (1989); Phys. Rev. B 41, 327 (1990). 
[12] C.A.R. Sa de Melo, M. Randeria, J.R. Engelbrecht, Phys. Rev. Lett. 71, 3202 (1993).

[13] R. Haussmann, Phys. Rev. B 49, 12975 (1994).

[14] J. Maly, B. Janko, K. Levin, Phys. Rev. B 59, 1354 (1999); Q. Chen, I. Kosztin, B. Janko, K. Levin, Phys. Rev. B 59, 7083 (1999); I. Kosztin, Q. Chen, Y. Kao, K. Levin, Phys. Rev. B 61, 11662 (2000).

[15] S. Schmitt-Rink, C.M. Varma, A.E. Ruckenstein, Phys. Rev. Lett. 63, 445 (1989).

[16] S.V. Traven, Phys. Rev. Lett. 73, 3451 (1994).

[17] M. Randeria, in: Bose-Einstein Condensation, Eds. A. Griffin, D. Snoke, S. Stringari, Cambridge University Press, Cambridge 1995, p. 355.

[18] R. Micnas, T. Kostyrko, in: Recent Progress in High Temperature Superconductivity, Lecture Notes in Physics, Eds. J. Klamut, B.W. Veal, B.M. Dabrowski, P.W. Klamut, M. Kazimierski, Vol. 475, Springer Verlag, Berlin 1996, p. 221, and refs. therein.

[19] J. Hubbard, Phys. Rev. Lett. 3, 77 (1959); R.L. Stratonovich, Sov. Phys. Doklady 2, 371 (1959).

[20] M. Drechsler, W. Zwerger, Ann. Phys. (Leipzig) 1, 15 (1992).

[21] P. Pieri, G.C. Strinati, Phys. Rev. B 61, 15370 (2000); F. Pistolesi, G.C. Strinati, ibid., 53, 15168 (1996); T.K. Kopeć, H. Umezawa, ibid., B 47, 8923 (1993).

[22] A. Tokumitu, K. Miyake, K. Yamada, Phys. Rev. B 47, 11988 (1993).

[23] R. Micnas, S. Robaszkiewicz, Phys. Rev. B 45, 9900 (1992).

[24] E.V. Gorbar, V.M. Loktev, S.G. Sharapov, ITP-95-6E preprint of the N.N. Bogolyubov Institute for Theoretical Physics, Kiev, Ukraine; Physica C 257, 355 (1966)

[25] W. Czart, S. Robaszkiewicz, Phys. Rev. B 64, 104511 (2001) and refs. therein.

[26] J.M. Luttinger, J.C. Ward, Phys. Rev. 118, 1417 (1960).

[27] G. Baym, Phys. Rev. 127, 1391 (1962).

[28] J.W. Serene, Phys. Rev. B 40, 10873 (1989).

[29] R. Micnas, M.H. Pedersen, S. Schafroth, T. Schneider, J.J. Rodriguez-Nunez, H. Beck, Phys. Rev. B 52, 16223 (1995); M.H. Pedersen, J.J. Rodriguez-Nunez, H. Beck, T. Schneider, S. Schafroth, Z. Phys. B 103, 21 (1997); see also J.J. Deisz, D.W. Hess, J.W. Serene, Phys. Rev. Lett. 80, 373 (1998); W. Keller, W. Metzner, U. Schollwock, Phys. Rev. B 60, 3449 (1999); D. Rohe, W. Metzner, Phys. Rev. B 63, 224509 (2001); B. Kyung, Phys. Rev. B 63, 014502 (2001).

[30] H.-U. Everts, Z. Phys. 199, 211 (1967).

[31] J.A. Hertz, M. Klenin, Phys. Rev. B 10, 1084 (1974).

[32] B.L. Gyorffy, J.B. Staunton, G.M. Stocks, Phys. Rev. B 44, 5190 (1991).

[33] K.A. Park, R. Joynt, Phys. Rev. B 48, 16833 (1993).

[34] T. Moriya, Spin Fluctuations in Itinerant Electron Magnetism, Springer Verlag, Berlin 1985, p. 153. 